\title{
Fecal Microbiota Transplantation: A Microbiome Modulation Technique for Alzheimer's Disease
}

\author{
Varsha Nandwana $^{1}$, Shibajee Debbarma ${ }^{2}$ \\ 1. Medicine, Lady Hardinge Medical College and Associated Hospitals, New Delhi, IND 2. Community Medicine, Lady \\ Hardinge Medical College and Associated Hospitals, New Delhi, IND
}

Corresponding author: Varsha Nandwana, varshanan3@gmail.com

\begin{abstract}
Alzheimer's disease (AD) is the most common form of dementia and the fifth leading cause of death among the elderly. $\mathrm{AD}$ involves parts of the brain that can lead to progressive memory loss and impaired language skills and cognitive thinking, affecting one's ability to carry out daily activities. Aging, bad dietary habits, family history, as well as altered gut microbiota composition may play a role in the pathogenesis of AD. Although the association between the imbalance of gut microbiota and $\mathrm{AD}$ is still difficult to determine, it has been suggested that dysbiosis can lead to the increased secretion of lipopolysaccharides and amyloid, which may impair the permeability of the intestine and the blood-brain barrier. Moreover, it can progress the process of neuroinflammation, amyloid-beta formation, and ultimately neuronal death. Microbiota-targeted interventions such as personalized diet, probiotics, or fecal microbiota transplantation (FMT) might represent a potential therapeutic option for $\mathrm{AD}$. This review article discusses the procedure of FMT and its possible side effects on the recipient's body. In addition, we review the role of FMT in the context of its application in various nervous system-related disorders (AD, Parkinson's disease, multiple sclerosis).
\end{abstract}

Categories: Family/General Practice, Neurology, Gastroenterology

Keywords: alzheimer's disease, gut microbiota, dysbiosis, fecal microbiota transplantation, gut-brain axis

\section{Introduction And Background}

The gut microbiota plays a key role in modulating the gut-brain axis, which is a bidirectional communication network that involves the central nervous system, the autonomic nervous system (sympathetic and parasympathetic branches), the enteric nervous system, and the hypothalamic-pituitary-adrenal axis [1]. Recent advances have revealed that the microbiota of the human gut has numerous beneficial functions, such as immune system development, resistance to pathogens, vitamin synthesis, production of metabolites such as short-chain fatty acids (SCFAs), nutrient and drug metabolism, and maintenance of the structural integrity of the intestinal mucosal barrier [2]. In humans, dysbiosis and changes in gut microbiome composition have been found to contribute to inflammatory bowel disease, type 2 diabetes, metabolic syndrome, obesity, colorectal cancer, Alzheimer's disease (AD), and numerous other diseases [3]. $\mathrm{AD}$ is a disastrous neurological disorder affecting 5.8 million Americans (aged 65 years or older) in 2020. This number is expected to increase to 14 million by 2060. As the prevalence of AD increases, the total estimated worldwide cost of dementia is also expected to increase to US $\$ 2.0$ trillion by 2030 [4]. AD was the sixth most common cause of death in 2017, accounting for 121,404 deaths in the United States, and the fifth most common cause of death among elderly Americans (65+ years) [5]. The sizeable economic burden of $\mathrm{AD}$, as well as its growing prevalence, are leading researchers to look for preventive or disease-modifying treatments. There are various gut microbiota modulation interventions such as diet modification, prebiotics, probiotics, synbiotics, or fecal microbiota transplantation (FMT). FMT includes the transplantation of the gut microbiota from a donor to a recipient to refurbish the intestinal microflora of the recipient. It has been proven to be a successful treatment for recurrent Clostridium difficile infections [6]. In this review, we summarize the procedure of FMT and its application in the treatment of various neurological disorders with a special emphasis on $\mathrm{AD}$.

\section{Review}

\section{Dysbiosis and Alzheimer's disease}

In Western countries, more than 40 million elderly people suffer from $\mathrm{AD}$, accounting for over $65 \%$ of all dementia cases. The increased life expectancy of the global population has led to an increase in age-related diseases, including $\mathrm{AD}$ [7]. The primary neuropathological criteria for $\mathrm{AD}$ diagnosis include the presence of misfolded amyloid-beta $(\mathrm{A} \beta$ ) plaques and deposition of hyperphosphorylated tau as neurofibrillary tangles (NFT) [8]. Although the greatest known risk factor for $\mathrm{AD}$ is increasing age, multiple factors such as genetics, family history, lifestyle, and environment may influence the onset of AD. There is increasing evidence suggesting the linkage between microbes that naturally reside in the body and the development of many of the hallmark features of $\mathrm{AD}$, including $\mathrm{A} \beta$ plaques and NFT [9]. It has been shown that the gut microbiota plays a crucial role in some brain processes such as myelinization, microglial activation, and neurogenesis, which is closely related to behavioral, mood, and cognitive modulation [10]. In addition to aging, unhealthy 
diet, stress, or obesity, several factors that affect gut microbiota composition are associated with $\mathrm{AD}$ [11]. The impaired gut microbial composition alters the expression of tight junction proteins, that is, zonulin and occludin, in epithelial colon cells. Intestinal permeability is increased due to problems in tight junction competence. The impaired gut microbial composition can increase lipopolysaccharides, amyloids, and trimethylamine N-oxide, and simultaneously decrease the beneficial metabolites such as SCFA and hydrogen [11]. Toll-like receptors (TLR) 2 and 4 are activated by exposure to enteropathogenic bacteria. This results in nuclear factor kappa B (NFkB) activation and secretion of pro-inflammatory cytokine interleukin-12 (IL-12). When IL-12 is increased, T-helper (Th)1/Th2 immune responses improve accompanied by an increase in tumor necrosis factor (TNF), interferon-gamma (IFN- $\gamma$ ), and IL-6 [12]. Consequently, elevated levels of circulating pro-inflammatory cytokines could enter the central nervous system through the leaky blood-brain barrier (BBB) (due to disrupted endothelial tight junction), leading to the translocation of blood-borne immune cells such as macrophages and neutrophils. In addition, as a result of inflammatory mediators such as cytokines and microbes, or bacterial products, microglia become activated, causing the breakdown of the extracellular matrix and dysfunction of astrocyte, pericyte, and neuronal cells [13]. This cascade acts as a trigger for $\mathrm{A} \beta$ and tau accumulation, leading to neurodegeneration and progression of $\mathrm{AD}$ pathology (Figure 1).

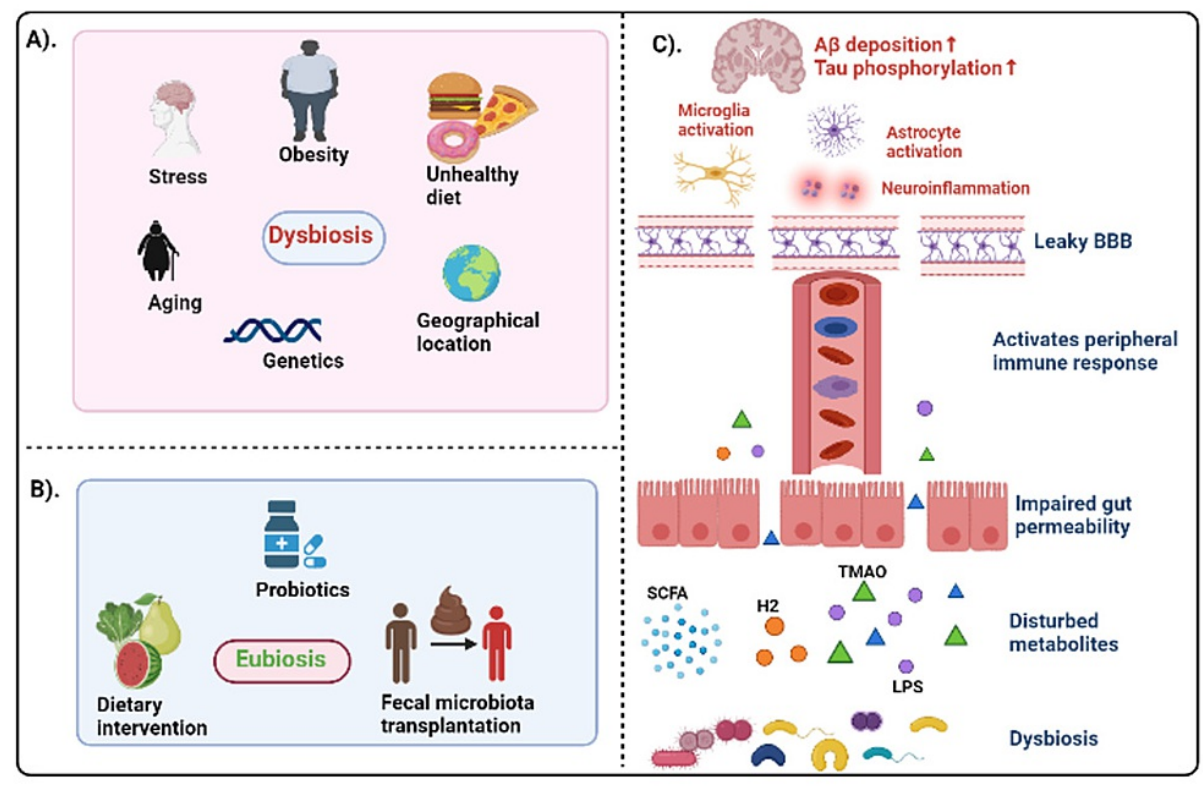

FIGURE 1: (A) Various factors influencing dysbiosis, that is, alteration of gut flora. (B) Various microbiota modulation techniques inducing eubiosis, that is, restoration of altered gut composition. (C) Schematic diagram representing the interplay between dysbiosis and $A D$ pathology.

Created with BioRender.com.

A $\beta$ : amyloid-beta; AD: Alzheimer's disease; BBB: blood-brain barrier; LPS: lipopolysaccharide; SCFA: shortchain fatty acid; TMAO: trimethylamine $\mathrm{N}$-oxide

During the course of recent research, Cattaneo et al. indicated that cognitive impairment and amyloid deposition in the brain was associated with an increase in the abundance of pro-inflammatory taxa Escherichia/Shigella and reduced anti-inflammatory taxon Eubacterium rectale [14]. In a healthy individual, two phyla, Firmicutes and Bacteroidetes, represent about 90\% of the gut microbiota, while the rest is composed of Actinobacteria, Proteobacteria, Fusobacteria, and Verrucomicrobia [15]. Vogt et al. examined fecal samples from $A D$ patients and compared them with controls (age and sex-matched individuals). According to the study, Firmicutes and Bifidobacterium are less abundant in the AD microbiome whereas Bacteroides are more abundant. In addition, they observed a correlation between the relative abundance of bacterial genera and the levels of cerebrospinal fluid biomarkers of AD [16]. According to Bäuerl et al., a transgenic APP/PS1 (Tg) mouse model was also successful in showing how AD pathology shifted gut bacterial profiles in favor of Proteobacteria and Erysipelotrichaceae, which potentially contributed to disease progression and severity [17]. Cox et al. demonstrated that Bacteroides colonization worsens A $\beta$ deposition, while long-term calorie restriction may alter the gut environment and inhibit the cultivation of microbes that contribute to age-related cognitive decline [18]. 


\section{Potential therapeutic strategies for Alzheimer's disease}

As it is already known that dysbiosis is strongly correlated with the development of neurodegenerative diseases, gut microbiota modification can also play a key role in combating AD. Most therapies for AD simply delay the loss of cognition and memory as there is no definitive treatment for AD. Several recent studies have pointed toward the role of the human microbiome in regulating multiple neurochemical pathways through the gut-brain axis [19]. It has been shown that plant-based diets, omega-3 polyunsaturated fatty acids, antioxidants, and reducing dietary saturated fats, animal-derived proteins, and refined sugars reduce the risk of cognitive decline and early onset of $\mathrm{AD}$ [20]. Diet-based therapeutic interventions include calorie restriction, prebiotic-rich diet, probiotic supplementation, probiotic-enriched diet, and polyphenol supplementation [19].

The probiotic bacterium helps promote the health of the recipient, while the prebiotic bacterium is primarily a food source for the probiotic bacterium [21]. A study by Bonfili et al. treated a transgenic mouse model of AD with a cocktail of Lactobacillus (L. acidophilus, L. brevis, L. delbrueckii subsp. bulgaricus, L. paracasei, $L$. plantarum) and Bifidobacteria (B. breve, B. longum, B. infantis) called SLAB 51 for a duration of four months. The increase in Bifidobacterium spp. and the reduction in Campylobacterales spp. observed in AD mice upon administration with SLAB51 as well as the altered content of metabolites of intestinal bacteria such as SCFA improved cognitive functions [22]. A study by Ho et al. reported that intestinal microbiota act to protect against $\mathrm{AD}$ in part by enhancing the formation of select SCFAs that inhibit the formation of soluble aggregates of $\mathrm{A} \beta$ [23]. In one study, Kobayashi et al. showed that $B$. breve $\mathrm{A} 1$ consumption decreased inflammatory and immune-reactive genes expressed in hippocampal neurons [21]. In a recent study, Abraham et al. showed that probiotics were sufficient to improve cognitive performance in mice with $\mathrm{AD}$ compared to mice not treated with probiotics [24].

In addition to dietary intervention and probiotics, FMT is the most effective therapeutic option that modulates the gut microbiome. This approach has shown improvements in various neurological disorders such as AD, Parkinson's disease (PD), and multiple sclerosis (MS).

\section{Fecal microbiota transplantation}

Historical Applications of Fecal Microbiota Transplantation

Approximately 1,700 years ago, when human fecal material was called "yellow soup," the concept of FMT was introduced by a Chinese medical scientist named Ge Hong when he administered the "soup" orally to treat patients with severe diarrhea or gastroenteritis [25]. Christian Paullini, a German-born scientist, was the first to demonstrate the curative potential of human excretion with his work on "Heilsame DreckApotheke," that is, "healing mud pharmacy” [26]. The first human fecal enemas were used to treat pseudomembranous colitis by Eiseman et al. in 1958 using four case studies [27]. Borody et al. performed "an exchange of bowel flora” in a patient with refractory ulcerative colitis showing full and lasting clinical recovery after treatment [28]. In 2013, van Nood et al. noticed that, in patients with recurrent $C$. difficile infection, the introduction of donor feces had more efficacy in comparison to antibiotics alone [29].

\section{Procedure for Fecal Microbiota Transplantation}

The donors for FMT can be selected from intimate partners, family members, or unrelated volunteers (Figure 2) [30]. The following conditions are generally considered to prevent donor recruitment: taking antibiotics within three months; being on immunosuppressive or chemotherapy agents; acquiring HIV or hepatitis B or $C$ recently; being obese, having inflammatory bowel disease (IBD) or irritable bowel syndrome; having an underlying infection, GI malignancy, polyposis, high-risk sexual behaviors, and use of illicit drugs; and having a history of recent incarceration or travel to areas with endemic diarrhea [31,32]. A potential donor with an acceptable medical history needs to be screened for infective diseases by undergoing blood and stool tests [33]. Nowadays, due to the coronavirus disease 2019 pandemic, it has been suggested to rule out severe acute respiratory syndrome coronavirus 2 infection in all donors through nasopharyngeal polymerase chain reaction and RNA detection in rectal swab [34]. A potential donor can provide fresh stool within one month after screening. The stool is collected and sealed in a clean opaque plastic bag before placing it in a larger storage bag [32]. Though the ideal amount of stool weight to be used for FMT has not been standardized, approximately 50 to $60 \mathrm{~g}$ of stool is recommended for each treatment. The stool can be stored for up to eight hours at $4^{\circ} \mathrm{C}$ as after eight hours at $4^{\circ} \mathrm{C}$ or room temperature, bacterial survival declines. The feces are dissolved in approximately $150 \mathrm{~mL}$ of normal saline by hand stirring or by using a blender [35]. The mixture is then filtered through a sterile gauze pad or steel strainers to remove large particulate matter [36]. Thereafter, the suspension is drawn into $60 \mathrm{~mL}$ catheter tip syringes. Furthermore, the final fecal material should be clearly labeled and stored at $-80^{\circ} \mathrm{C}$. On the day of the infusion, it should be thawed in a warm water bath at $37^{\circ} \mathrm{C}$ and infused within four hours from thawing [32] (Figure 2).

FMT can be administered directly to the colon using colonoscopy (efficacy of 84-93\%) [37] and less frequently through flexible sigmoidoscopy or an enema [38]. For patients with ileus, severe colitis, or contraindication to colonoscopy, FMT can be provided through the upper GI tract via nasoenteric tubes, esophagogastroduodenoscopy, or capsule ingestion [38] (Figure 2). Capsule delivery is the most recent 
modality of FMT, which seems to be favorable for those who are opposed to lower GI tract access and are geographically distant from an institution that performs colonoscopy [39]. Before an FMT, the patient must be vigorously prepared so that no residual stool is present [35]. If the fecal solution is administered via colonoscopy, the recipient usually undergoes a bowel preparation to flush out the pre-existing bacteria. When a nasogastric tube is used to administer the fecal sample, a proton pump inhibitor may be administered in advance to increase the survival of the transplanted bacteria [40] (Figure 2).

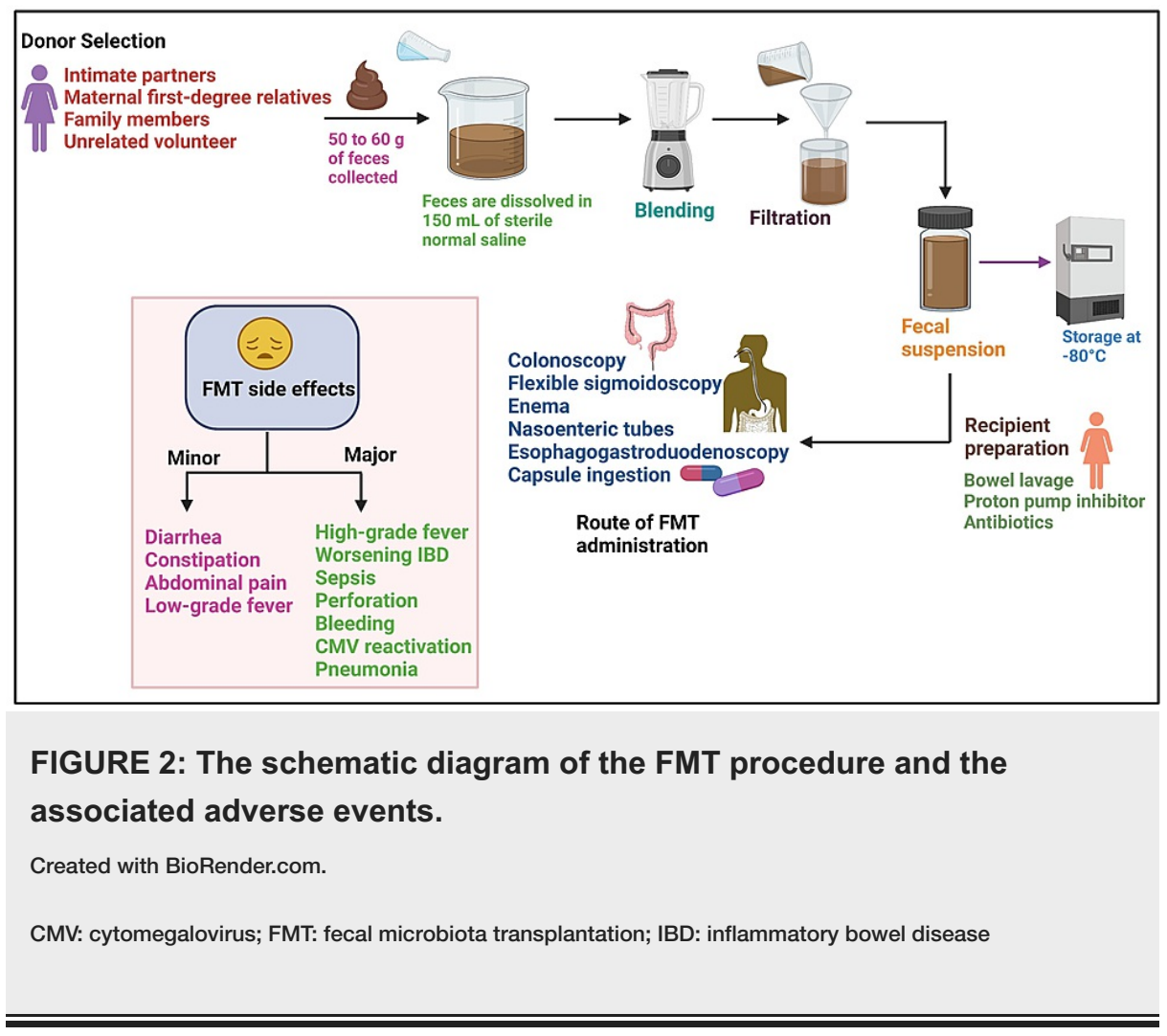

Adverse Events of Fecal Microbiota Transplantation

In general, FMT is a safe and efficacious therapeutic method with limited side effects. Many recent large studies have confirmed that minor adverse events including diarrhea, constipation, abdominal discomfort, bloating, and transient low-grade fevers are the most likely to occur and are likely to resolve within days to weeks. Uncommon severe side effects such as high-grade fever, worsening IBD activity, infection and sepsis, the transmission of enteric pathogens, perforation, bleeding, cytomegalovirus reactivation, and pneumonia [41] are associated with endoscopy and sedation (Figure 2). It has been reported in some case reports that there might be some abeyant connection between FMT and certain conditions such as rheumatoid arthritis, idiopathic thrombocytopenic purpura, and peripheral neuropathy [42]. FMT recipients should be informed about the potential adverse effects before the procedure.

\section{Fecal microbiota transplantation as a restorer of altered gut microbiota in Alzheimer's disease}

Restoring gut microbial composition and reducing dysbiosis by FMT could be an effective approach for various neurological disorders, but the evidence is limited. In an experiment conducted by Zhan et al., mice were first treated with broad-spectrum antibiotics for 14 consecutive days. Subsequently, the authors transplanted fecal bacteria from senescence-accelerated mouse prone 8 (SAMP8) or senescence-accelerated mouse resistant 1 (SAMR1) mice into pseudo-germ-free mice and noticed that pseudo-germ-free mice that received fecal bacteria transplants from SAMR1 mice but not from SAMP8 mice showed improvements in spatial learning and memory [43]. Yu et al. used diabetic mice with and without cognitive deterioration and performed fecal bacteria transplantation from noncognitive dysfunction mice into the gut of pseudo-germfree mice. They noticed a significant improvement in host Morris water maze performance indices, an effect linked with change in $\beta$-diversity and relative abundance of host intestinal microbes [44]. Furthermore, Dodiya et al. demonstrated that fecal microbiota transplantation from age- and sex-matched $\mathrm{AD}$ male mice without antibiotics into antibiotic-treated AD male mice reinstituted the intestinal bacteria and partially refurbished $A \beta$ pathology and microglial morphology. They observed relatively higher expression of antiinflammatory cytokines such as IL-10 and lower expression of pro-inflammatory cytokines including IL-1B, IL-2, IL-3, IL-17A, LIX (CXC5), RANTES (CCL5), and the cluster of differentiation 30 (CD30) and CD40 in antibiotic-treated male mice. [45]. Fuji et al. created a humanized mice model by transplanting germ-free 
mice with a fecal sample from a healthy person and an $\mathrm{AD}$ patient. They noticed that gut microbiota transplanted from an affected patient affected mouse behavior remarkably. Moreover, lower metabolites related to the nervous system, including $\gamma$-aminobutyrate, taurine, and valine, in the feces of mice transplanted with microbiota from $\mathrm{AD}$ patients has been noted [46]. Recently, using APPswe/PS1dE9 transgenic ( $\mathrm{Tg}$ ) mouse model, Sun et al. showed that FMT can reduce the brain deposition of A $\beta$ and decrease the phosphorylation of tau protein and levels of $A \beta 40$ and $A \beta 42$. In addition, improvement in cognitive function and an increase in synaptic plasticity have also been observed in Tg mice, thus demonstrating FMT as a potential therapeutic strategy for AD [47]. The case of an 82-year-old AD patient was reported by Hazan in 2020, who received a single FMT from his 85-year-old wife, but his symptoms of $C$. difficile infection resolved within two months following the test. In addition, after two months of FMT, the Mini-Mental State Examination (MMSE) score of the patient increased from 20 (mild cognitive impairment) to 26 (normal cognitive function), which further improved (MMSE score 29) after four months of follow up [48]. Holsinger et al. using the 5XFAD mouse model described an improvement in spatial and recognition memory and reduction in amyloid pathology after seven days of FMT from wild-type donor to 5XFAD mouse, thus exhibiting the efficacy of FMT as a therapeutic in AD [49].

\section{Fecal microbiota transplantation in various other neurological disorders}

PD is a progressive nervous system disorder that affects predominately dopamine-producing neurons in a specific area of the brain called substantia nigra. PD patients may experience resting tremor, bradykinesia, limb rigidity, gait, and balance problems. Sun et al. demonstrated that gut microbial dysbiosis is involved in PD pathogenesis using a PD mouse model. FMT from healthy mice improved motor function, increased striatal neurotransmitters, and decreased neuroinflammation [50]. Another study by Zhou et al. noticed increased dopamine levels in PD mice that had received FMT from normal mice with fasting-mimicking diet treatment to antibiotic-pretreated PD mice [51]. MS is a demyelinating disorder in which the immune system attacks the protective covering of nerves, thus disrupting the communication between the brain and the body. Patients with secondary progressive MS as well as concomitant recurrent $C$. difficile infections were treated with a single FMT. The FMT resolved the recurrent $C$. difficile infections and was suggested to prevent MS disease progression for over 10 years [52]. Li et al. conducted a study using experimental autoimmune encephalomyelitis, a mouse model of MS. They observed that FMT can correct altered gut microbiota and confer protection on the BBB, myelin, and axons, as well as can reduce the activation of microglia and astrocytes, thus suggesting the role of FMT as a potentially effective treatment for MS [53].

\section{Conclusions}

The composition of healthy gut microbiota may change due to nutrition, medications, lifestyle, geographical location, stress, and dietary habits. An imbalance in the intestinal bacteria can influence the onset and progression of $\mathrm{AD}$. Thus, modulation of gut microbiota using dietary intervention, probiotics, and FMT represents a forward-looking approach for various neurological disorders such as AD, PD, and MS. Currently, the rationale for the clinical application of FMT is based on a small number of case reports and animal models. Increasing evidence suggests that the capsular form containing desirable bacteria is a more comfortable option for patients and may replace FMT in the future. For a complete understanding of the role of FMT in neurological diseases, we need a large-scale, randomized, double-blind trial.

\section{Additional Information}

\section{Disclosures}

Conflicts of interest: In compliance with the ICMJE uniform disclosure form, all authors declare the following: Payment/services info: All authors have declared that no financial support was received from any organization for the submitted work. Financial relationships: All authors have declared that they have no financial relationships at present or within the previous three years with any organizations that might have an interest in the submitted work. Other relationships: All authors have declared that there are no other relationships or activities that could appear to have influenced the submitted work.

\section{Acknowledgements}

We would like to thank Dr. Nitesh K. Nandwana (Ph.D.) for his assistance in preparing this review article.

\section{References}

1. Carabotti M, Scirocco A, Maselli MA, Severi C: The gut-brain axis: interactions between enteric microbiota, central and enteric nervous systems. Ann Gastroenterol. 2015, 28:203-9.

2. Quigley EM: Gut bacteria in health and disease. Gastroenterol Hepatol (N Y). 2013, 9:560-9.

3. Bekkering P, Jafri I, van Overveld FJ, Rijkers GT: The intricate association between gut microbiota and development of type 1, type 2 and type 3 diabetes. Expert Rev Clin Immunol. 2013, 9:1031-41. 10.1586/1744666X.2013.848793

4. World Alzheimer Report 2015. The global impact of dementia: an analysis of prevalence, incidence, cost and trends. (2015). Accessed: June 07, 2021: https://www.alzint.org/resource/world-alzheimer-report-2015/.

5. Gaugler J, James B, Johnson T, Marin A, Weuve J: 2019 Alzheimer's disease facts and figures. Alzheimer Dement. 2019, 15:321-87. 10.1016/j.jalz.2019.01.010 
6. Borody TJ, Brandt LJ, Paramsothy S: Therapeutic faecal microbiota transplantation: current status and future developments. Curr Opin Gastroenterol. 2014, 30:97-105. 10.1097/MOG.0000000000000027

7. Giovannini MG, Lana D, Traini C, Vannucchi MG: The microbiota-gut-brain axis and Alzheimer disease. From dysbiosis to neurodegeneration: focus on the central nervous system glial cells. J Clin Med. 2021, 10:2358. 10.3390/jcm10112358

8. Tiwari S, Atluri V, Kaushik A, Yndart A, Nair M: Alzheimer's disease: pathogenesis, diagnostics, and therapeutics. Int J Nanomedicine. 2019, 14:5541-54. 10.2147/IJN.S200490

9. Bulgart HR, Neczypor EW, Wold LE, Mackos AR: Microbial involvement in Alzheimer disease development and progression. Mol Neurodegener. 2020, 15:42. 10.1186/s13024-020-00378-4

10. Cenit MC, Sanz Y, Codoñer-Franch P: Influence of gut microbiota on neuropsychiatric disorders. World J Gastroenterol. 2017, 23:5486-98. 10.3748/wig.v23.i30.5486

11. Janeiro MH, Ramírez MJ, Solas M: Dysbiosis and Alzheimer's disease: cause or treatment opportunity? [In Press]. Cell Mol Neurobiol. 2021, 10.1007/s10571-020-01024-9

12. Ganesh BP, Versalovic J: Luminal conversion and immunoregulation by probiotics . Front Pharmacol. 2015, 6:269. 10.3389/fphar.2015.00269

13. Parker A, Fonseca S, Carding SR: Gut microbes and metabolites as modulators of blood-brain barrier integrity and brain health. Gut Microbes. 2020, 11:135-57. 10.1080/19490976.2019.1638722

14. Cattaneo A, Cattane N, Galluzzi S, et al.: Association of brain amyloidosis with pro-inflammatory gut bacterial taxa and peripheral inflammation markers in cognitively impaired elderly. Neurobiol Aging. 2017, 49:60-8. 10.1016/j.neurobiolaging.2016.08.019

15. Glass P: The gut microbiome \& its value in autoimmune diseases . Baylor University, Waco, TX; 2021.

16. Vogt NM, Kerby RL, Dill-McFarland KA, et al.: Gut microbiome alterations in Alzheimer's disease . Sci Rep. 2017, 7:13537. 10.1038/s41598-017-13601-y

17. Bäuerl C, Collado MC, Diaz Cuevas A, Viña J, Pérez Martínez G: Shifts in gut microbiota composition in an APP/PSS1 transgenic mouse model of Alzheimer's disease during lifespan. Lett Appl Microbiol. 2018, 66:464-71. 10.1111/lam.12882

18. Cox LM, Schafer MJ, Sohn J, Vincentini J, Weiner HL, Ginsberg SD, Blaser MJ: Calorie restriction slows agerelated microbiota changes in an Alzheimer's disease model in female mice. Sci Rep. 2019, 9:17904. 10.1038/s41598-019-54187-x

19. Bonfili L, Cecarini V, Gogoi O, et al.: Microbiota modulation as preventative and therapeutic approach in Alzheimer's disease. FEBS J. 2021, 288:2836-55. 10.1111/febs.15571

20. Pistollato F, Iglesias RC, Ruiz R, et al.: Nutritional patterns associated with the maintenance of neurocognitive functions and the risk of dementia and Alzheimer's disease: a focus on human studies. Pharmacol Res. 2018, 131:32-43. 10.1016/j.phrs.2018.03.012

21. Kobayashi Y, Sugahara H, Shimada K, et al.: Therapeutic potential of Bifidobacterium breve strain A1 for preventing cognitive impairment in Alzheimer's disease. Sci Rep. 2017, 7:13510. 10.1038/s41598-017-133682

22. Bonfili L, Cecarini V, Berardi S, et al.: Microbiota modulation counteracts Alzheimer's disease progression influencing neuronal proteolysis and gut hormones plasma levels. Sci Rep. 2017, 7:2426. 10.1038/s41598017-02587-2

23. Ho L, Ono K, Tsuji M, Mazzola P, Singh R, Pasinetti GM: Protective roles of intestinal microbiota derived short chain fatty acids in Alzheimer's disease-type beta-amyloid neuropathological mechanisms. Expert Rev Neurother. 2018, 18:83-90. 10.1080/14737175.2018.1400909

24. Abraham D, Feher J, Scuderi GL, et al.: Exercise and probiotics attenuate the development of Alzheimer's disease in transgenic mice: role of microbiome. Exp Gerontol. 2019, 115:122-31. 10.1016/j.exger.2018.12.005

25. Zhang F, Luo W, Shi Y, Fan Z, Ji G: Should we standardize the 1,700-year-old fecal microbiota transplantation?. Am J Gastroenterol. 2012, 107:1755. 10.1038/ajg.2012.251

26. Tetro J, Allen-Vercoe E: The human microbiome handbook. DEStech Publications, Inc., Lancaster, PA; 2016.

27. Eiseman B, Silen W, Bascom GS, Kauvar AJ: Fecal enema as an adjunct in the treatment of pseudomembranous enterocolitis. Surgery. 1958, 44:854-9.

28. Borody TJ, George L, Andrews P, et al.: Bowel-flora alteration: a potential cure for inflammatory bowel disease and irritable bowel syndrome?. Med J Aust. 1989, 150:604. 10.5694/j.1326-5377.1989.tb136704.x

29. van Nood E, Vrieze A, Nieuwdorp M, et al.: Duodenal infusion of donor feces for recurrent Clostridium difficile. N Engl J Med. 2013, 368:407-15. 10.1056/NEJMoa1205037

30. Bakken JS, Borody T, Brandt LJ, et al.: Treating Clostridium difficile infection with fecal microbiota transplantation. Clin Gastroenterol Hepatol. 2011, 9:1044-9. 10.1016/j.cgh.2011.08.014

31. Woodworth MH, Carpentieri C, Sitchenko KL, Kraft CS: Challenges in fecal donor selection and screening for fecal microbiota transplantation: a review. Gut Microbes. 2017, 8:225-37. 10.1080/19490976.2017.1286006

32. Nicco C, Paule A, Konturek P, Edeas M: From donor to patient: collection, preparation and cryopreservation of fecal samples for fecal microbiota transplantation. Diseases. 2020, 8:9. 10.3390/diseases8020009

33. Bibbò S, Settanni CR, Porcari S, Bocchino E, Ianiro G, Cammarota G, Gasbarrini A: Fecal microbiota transplantation: screening and selection to choose the optimal donor. J Clin Med. 2020, 9:1757. 10.3390/jcm9061757

34. Ianiro G, Mullish BH, Kelly CR, et al.: Screening of faecal microbiota transplant donors during the COVID-19 outbreak: suggestions for urgent updates from an international expert panel. Lancet Gastroenterol Hepatol. 2020, 5:430-2. 10.1016/S2468-1253(20)30082-0

35. Fecal transplantation for C difficile: a how-to guide. (2013). Accessed: June 07, 2021: https://www.medscape.com/viewarticle/779307.

36. Wang JW, Kuo CH, Kuo FC, et al.: Fecal microbiota transplantation: review and update. J Formos Med Assoc. 2019, 118 Suppl 1:S23-31. 10.1016/j.jfma.2018.08.011

37. Kassam Z, Lee CH, Yuan Y, Hunt RH: Fecal microbiota transplantation for Clostridium difficile infection: systematic review and meta-analysis. Am J Gastroenterol. 2013, 108:500-8. 10.1038/ajg.2013.59

38. Allegretti JR, Kassam Z, Osman M, Budree S, Fischer M, Kelly CR: The 5D framework: a clinical primer for 
fecal microbiota transplantation to treat Clostridium difficile infection. Gastrointest Endosc. 2018, 87:18-29. 10.1016/j.gie.2017.05.036

39. Zipursky JS, Sidorsky TI, Freedman CA, Sidorsky MN, Kirkland KB: Patient attitudes toward the use of fecal microbiota transplantation in the treatment of recurrent Clostridium difficile infection. Clin Infect Dis. 2012, 55:1652-8. 10.1093/cid/cis809

40. Matsuoka K, Mizuno S, Hayashi A, Hisamatsu T, Naganuma M, Kanai T: Fecal microbiota transplantation for gastrointestinal diseases. Keio J Med. 2014, 63:69-74. 10.2302/kjm.2014-0006-RE

41. Dailey FE, Turse EP, Daglilar E, Tahan V: The dirty aspects of fecal microbiota transplantation: a review of its adverse effects and complications. Curr Opin Pharmacol. 2019, 49:29-33. 10.1016/j.coph.2019.04.008

42. Brandt LJ, Aroniadis OC, Mellow M, et al.: Long-term follow-up of colonoscopic fecal microbiota transplant for recurrent Clostridium difficile infection. Am J Gastroenterol. 2012, 107:1079-87. 10.1038/ajg.2012.60

43. Zhan G, Yang N, Li S, et al.: Abnormal gut microbiota composition contributes to cognitive dysfunction in SAMP8 mice. Aging (Albany NY). 2018, 10:1257-67. 10.18632/aging.101464

44. Yu F, Han W, Zhan G, et al.: Abnormal gut microbiota composition contributes to cognitive dysfunction in streptozotocin-induced diabetic mice. Aging (Albany NY). 2019, 11:3262-79. 10.18632/aging.101978

45. Dodiya HB, Kuntz T, Shaik SM, et al.: Sex-specific effects of microbiome perturbations on cerebral A $\beta$ amyloidosis and microglia phenotypes. J Exp Med. 2019, 216:1542-60. 10.1084/jem.20182386

46. Fujii Y, Nguyen TT, Fujimura Y, Kameya N, Nakamura S, Arakawa K, Morita H: Fecal metabolite of a gnotobiotic mouse transplanted with gut microbiota from a patient with Alzheimer's disease. Biosci Biotechnol Biochem. 2019, 83:2144-52. 10.1080/09168451.2019.1644149

47. Sun J, Xu J, Ling Y, et al.: Fecal microbiota transplantation alleviated Alzheimer's disease-like pathogenesis in APP/PS1 transgenic mice. Transl Psychiatry. 2019, 9:189. 10.1038/s41398-019-0525-3

48. Hazan S: Rapid improvement in Alzheimer's disease symptoms following fecal microbiota transplantation: a case report. J Int Med Res. 2020, 48:300060520925930. 10.1177/0300060520925930

49. Holsinger RD, Elangovan S: Neuroprotective effects of fecal microbiota transplantation in a mouse model of Alzheimer's disease: development of new models and analysis methods/validation of pre-clinical methods. Alzheimer Dement. 2020, 16:e046523. 10.1002/alz.046523

50. Sun MF, Zhu YL, Zhou ZL, et al.: Neuroprotective effects of fecal microbiota transplantation on MPTPinduced Parkinson's disease mice: gut microbiota, glial reaction and TLR4/TNF- $\alpha$ signaling pathway. Brain Behav Immun. 2018, 70:48-60. 10.1016/j.bbi.2018.02.005

51. Zhou ZL, Jia XB, Sun MF, et al.: Neuroprotection of fasting mimicking diet on MPTP-induced Parkinson's disease mice via gut microbiota and metabolites. Neurotherapeutics. 2019, 16:741-60. 10.1007/s13311-01900719-2

52. Makkawi S, Camara-Lemarroy C, Metz L: Fecal microbiota transplantation associated with 10 years of stability in a patient with SPMS. Neurol Neuroimmunol Neuroinflamm. 2018, 5:e459. 10.1212/NXI.0000000000000459

53. Li K, Wei S, Hu L, et al.: Protection of fecal microbiota transplantation in a mouse model of multiple sclerosis. Mediators Inflamm. 2020, 2020:2058272. 10.1155/2020/2058272 